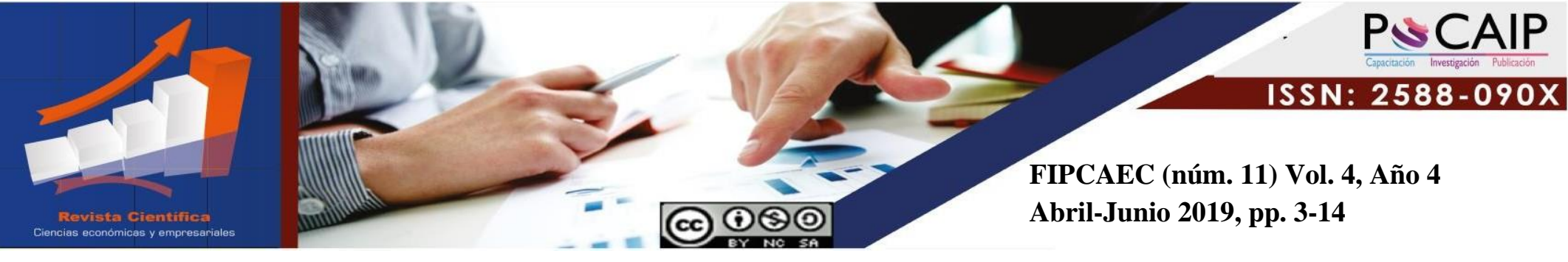

DOI: $10.23857 /$ fipcaec.v4i11.42

\title{
Indicadores para valorar la imagen pública externa del sector eléctrico en Esmeraldas
}

Indicators to assess the external public image of the electricity sector in Emeralds

Indicadores para avaliar a imagem pública externa do setor elétrico em Esmeraldas

\author{
Maritza Elena Montaño-Mina ${ }^{1}$ \\ liquenamm@hotmail.com \\ https://orcid.org/0000-0002-3721-4841
}

Correspondencia: liquenamm@hotmail.com

Recepción: 10/01/2019 * Aceptación: 02/03/2019 * Publicación: 15 /04/2019

${ }^{1}$ Ingeniera Comercial, Investigadora Independiente, Esmeralda, Ecuador. 


\title{
Resumen
}

Este artículo, siguió como objetivo estudiar los indicadores para valorar la imagen pública externa del sector eléctrico en Esmeraldas. Subraya una metodología con una orientación cuantitativa y descriptiva. La población fue de 220 usuarios del servicio eléctrico de Esmeraldas, se logró una muestra de 180 sujetos. El instrumento fue un cuestionario estructurado con una escala de 5 alternativas lickerd y como técnica la encuesta, administrada individualmente a los usuarios. En sus resultados se pudo apreciar que el 50\% de los investigados están en desacuerdo entre lo dicen que hace la empresa y el servicio real que prestan y dentro de sus conclusiones se develo que están en desacuerdo con una correcta imagen pública externa de la empresa eléctrica. Palabras clave: Imagen pública; empresa eléctrica; nivel de conocimiento; nivel de coincidencia e imagen corporativa.

\begin{abstract}
This article, continued to study the indicators to evaluate the external public image of the electricity sector in Esmeraldas. It underlines a methodology with a quantitative and descriptive orientation. The population was 220 users of the electric service of Esmeraldas, a sample of 180 subjects was achieved. The instrument was a structured questionnaire with a scale of 5 licked alternatives and as a technique the survey, administered individually to users. Its results could be seen that $50 \%$ of the users investigated disagree between what they say the company does and the actual service they provide and within their conclusions it is clear that they disagree with a correct external public image of the company Electric.
\end{abstract}

Keywords: public image; Power Company; level of knowledge; level of coincidence and corporate image.

\section{Resumo}

Este artigo continuou estudando os indicadores para avaliar a imagem pública externa do setor elétrico em Esmeraldas. Sublinha uma metodologia com uma orientação quantitativa e descritiva. A população era de 220 usuários do serviço elétrico de Esmeraldas, uma amostra de 180 sujeitos foi alcançada. $\mathrm{O}$ instrumento foi um questionário estruturado, com uma escala de 5 alternativas 
de lickerd e, como técnica, a pesquisa, administrada individualmente aos usuários. Em seus resultados, observou-se que 50\% dos investigados discordam entre o que dizem que a empresa faz e o serviço real que prestam e, dentro de suas conclusões, é desenvolvido que discordam de uma imagem pública externa correta da empresa elétrica.

Palavras-chave: Imagem pública; companhia elétrica; nível de conhecimento; nível de coincidência e imagem corporativa.

\section{Introducción}

La heterogeneidad que identifica a las organizaciones, globalmente, debido a su variada clasificación con respecto a tamaño, estructura y objetivos a alcanzar en la sociedad, debe ser plenamente conocida -desde un amplio panorama- por la gerencia para darle una adecuada aplicación. Entre esta clasificación se encuentran: Organizaciones según sus fines (con fines de lucro y sin fines de lucro), organizaciones según su formalidad (organizaciones formales e informales) y organizaciones según su grado de centralización (centralizadas o descentralizadas). Chiavenato (2006).

La organización, en los últimos tiempos y de manera global, se identifica como un sistema abierto porque participa activamente, en forma dinámica, cambiante y nunca en forma estática. Esta teoría confirma la existencia de la organización como un conjunto y ratifica su relación con el medio que lo circunda (Fernández, 2005), siendo necesario que adopte las mejores estrategias con la finalidad de generar un buen servicio al cliente, tal como lo proponen Aldana \& Piña (2017).

En las organizaciones que prestan servicios públicos, tal es el caso de la compañía eléctrica en Esmeraldas, existe un inadecuado direccionamiento de su imagen pública, sin lograr enlazar propósitos y oportunidades, al hacer uso de una trasmisión tradicional. Con base en lo expuesto, en este artículo se estudiaron los indicadores de conocimiento, coincidencia e imagen corporativa de la empresa eléctrica del cantón Esmeraldas. 


\section{Desarrollo}

La imagen,según Mouriz (2007), es producto de la interacción entre la imagen ideal que posee un determinado público de una organización o servicio y las características que percibe de la organización que la hacen más o menos capaz de satisfacer estas necesidades y por tanto, los acercan o los alejan de ese ideal. Mientras la imagen externa son todas aquellas características de la organización que son identificadas y evaluadas (positiva o negativamente) de manera semejante por el público externo.

Entre los indicadores que, Según Mouriz (2007), permiten valorar la imagen pública externa se encuentran:

Nivel de conocimiento: que tiene el público sobre la organización y su producto o servicio. Al respecto, Vilcarromero (2009, expone el conocimiento adquiere su razón de ser en el momento en que sirve para predecir, para describir, accionar o para orientar al hombre, generando insumos útiles. El conocimiento está en las personas, siendo deseable que tal conocimiento, se posicione en la organización como parte de su imagen, ofreciendo valor agregado en términos de reputación, cobertura y posicionamiento.

Nivel de coincidencia: está dado entre la imagen ideal y real que tiene el público acerca de organización y su producto o servicio. Gutiérrez (2011), considera que el nivel de coincidencia permite un enfoque global y correcta imagen corporativa, consiste en que esta se configure en la mente del público de forma que no experimente desviaciones con la identidad a la que la institución que la proyecte pretenda asociarse. Esto se obtendrá, fundamentalmente, al lograr que la empresa haga lo que dice y la influencia entre los diferentes públicos sobre lo que hace y dice que hace, refleje la mayor unicidad posible.

La imagen corporativa: se refiere las relaciones entre las imágenes pública e interna. La organización debe lograr que estos dos tipos de imagen estén en estrecha relación. Es importante aclarar que la interconexión entre estos dos tipos de imágenes es un resultado de los esfuerzos sistemáticos y conscientes por parte de la organización y que no se da fuera e independiente de la voluntad de esta, a la manera de las leyes o las regularidades. La imagen corporativa constituye la 
valoración cualitativa que hace un público determinado acerca de la cultura de una organización y de sus diferentes elementos.

Según Alba (2006), la imagen corporativa se refiere a cómo se percibe una empresa. Es decir, todo aquello que representa la significación y valores de una marca, refiere el logo, pero también elementos verbales, el diseño gráfico, sus diferentes elementos, y el componente psicológico, encargado de crear un vínculo emocional con el cliente, a través de valores culturales compartidos. Es evidente que la comunicación externa genera una imagen positiva de la organización, la cual fomenta en los empleados el orgullo de permanecer a la organización, es decir, crear un sentimiento de permanencia, que genera una imagen identitaria en la organización.

\section{Estrategias de comunicación que gestionan al público externo}

Gestionar la imagen externa positiva en una organización, proyecta un mensaje de identidad que se difunde a través de diferentes medios y va conformando un clima de respeto, identidad y marca representativa de la institución. En efecto, la comunicación externa es un proceso sistemático con un propósito: optimizar, generar o mantener las relaciones con los diferentes segmentos y actores de negocio, para ello se debe acordar una línea, que configure una imagen positiva de la marca. Lo planteado va configurando una imagen que se potencia a través de los mensajes publicitarios o comerciales y la comunicación externa, centrando el público objetivo. (Sánchez y col, 2009).

Entre los tipos de estrategias de comunicación que gestiona lo público externo, según Hernández, M. (2014), se encuentran:

- Comunicación externa estratégica: Consiste en conocer los datos de la competencia, las variables económicas y su evolución, así como los cambios en la legislación laboral, con el fin de alcanzar una posición competitiva en el mercado. Tiene por finalidad enterarse de los posibles datos de la competencia, la evolución de las variables en el mercado en relación a los objetivos. 
- Comunicación externa operativa: Es aquella que se realiza para el desenvolvimiento diario de la actividad empresarial, se efectúa con todos los públicos externos de la compañía: clientes y proveedores. Se utiliza para el desarrollo diario de la actividad de la empresa, y es la más importante a nivel de conocer, ya que es la que lleva todas las comunicaciones públicas externas de la empresa, tanto con proveedores, clientes, competidores y administradores.

- Comunicación externa de notoriedad: Su finalidad es mostrarla como una institución que informa dando a conocer sus productos y mejorar su imagen, entre otras. Las formas de darlo a conocer serían mediante la publicidad y la promoción. Pretende dar información a la empresa, tanto en mejorar la imagen como promocionar los productos. Es la que se encarga de la promoción, marketing, publicidad, patrocinios y otras actividades que hacen conocida la empresa de cara al exterior.

Todas estas formas de comunicación externa buscan un mismo objetivo, velar por el bien común de la organización, para ello da a conocer de manera amplia la imagen y productos, para posicionar una identidad, independientemente de las acciones de comunicación externa. Sin duda las estrategias de comunicación son de fundamental importancia para consolidar la imagen corporativa.

\section{Metodología}

El artículo se presenta con una metodología descriptiva y un enfoque cuantitativo (Hernández y col, 2014). La población estuvo constituida por 220 usuarios del servicio eléctrico de Esmeraldas, de los cuales 180 contestaron todas las preguntas, a partir de un muestro no probabilístico. Para la recolección de información se utilizó la técnica de la entrevista estructurada (Tamayo, 2012). La construcción del instrumento utilizado, se realizó a partir de las indagaciones de las teorías que evaluaran los indicadores de la imagen pública externa de una organización, la construcción del instrumento siguió las características de la población a encuestar y lod datos aportados permitieron alcanzar el objetivo propuesto. 


\section{Resultados}

La presentación de los resultados, es producto del análisis descriptivo frecuencial de los datos que permitieron evaluar los indicadores para valorar la imagen pública externa del sector eléctrico en Esmeraldas.

Tabla 1. Análisis descriptivo del indicador Nivel de conocimiento

\begin{tabular}{ccccc}
\hline & $\boldsymbol{F R}$ & $\%$ & $\boldsymbol{F R A}$ & $\% \boldsymbol{A}$ \\
\hline Totalmente de acuerdo & 24 & 13 & 24 & 13 \\
\hline De acuerdo & 36 & 20 & 60 & 33 \\
\hline Ni de acuerdo ni en desacuerdo & 72 & 40 & 132 & 73 \\
\hline En desacuerdo & 36 & 20 & 168 & 93 \\
\hline Totalmente en desacuerdo & 12 & 7 & 180 & 100 \\
\hline
\end{tabular}

Fuente: Elaboración propia (2019)

Gráfico 1. Análisis descriptivo del indicador Nivel de conocimiento

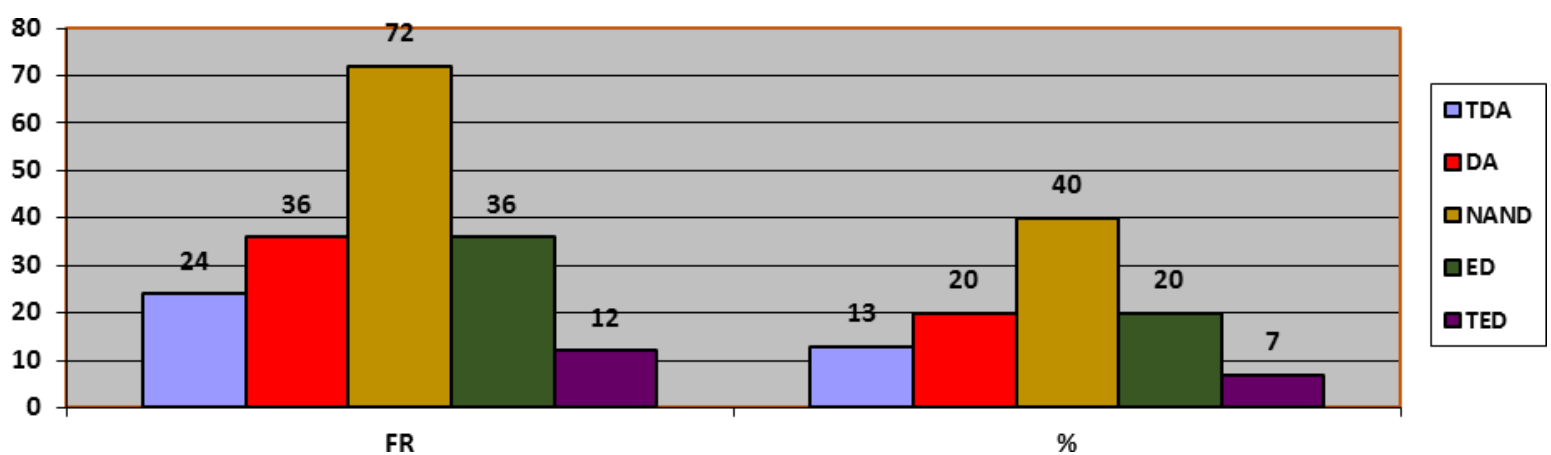

Fuente: Elaboración propia (2019)

En la tabla y gráfico 1, se presenta el análisis descriptivo del indicador nivel de conocimiento que tiene el público sobre la empresa eléctrica del Esmeraldas, en la que se indica que el $40 \%$ del total de los usuarios encuestados coincidieron en opinar que ni estaban de acuerdo ni en desacuerdo en conocer la organización que les permita predecir, describir, accionar o orientarlos en relación al servicio que prestan. Seguido del $20 \%$ por igual que consideraron 
estar en desacuerdo y de acuerdo. Los menores porcentajes se indicaron para las alternativas totalmente de acuerdo y totalmente en desacuerdo con el $13 \%$ y $7 \%$ respectivamente.

Tabla 2. Análisis Descriptivo del Indicador Nivel de Coincidencia

\begin{tabular}{ccccc}
\hline ANÁLISIS/ CRITERIOS & $\boldsymbol{F R}$ & $\%$ & $\boldsymbol{F R A}$ & $\% \boldsymbol{A}$ \\
\hline Totalmente de acuerdo & 18 & 10 & 18 & 10 \\
\hline De acuerdo & 30 & 17 & 48 & 27 \\
\hline $\begin{array}{c}\text { Ni de acuerdo ni en } \\
\text { desacuerdo }\end{array}$ & 42 & 23 & 90 & 50 \\
\hline En desacuerdo & 90 & 50 & 180 & 100 \\
\hline Totalmente en desacuerdo & 0 & 0 & 180 & 100 \\
\hline
\end{tabular}

Fuente: Elaboración propia (2019)

Gráfico 2. Análisis descriptivo del indicador Nivel de Coincidencia

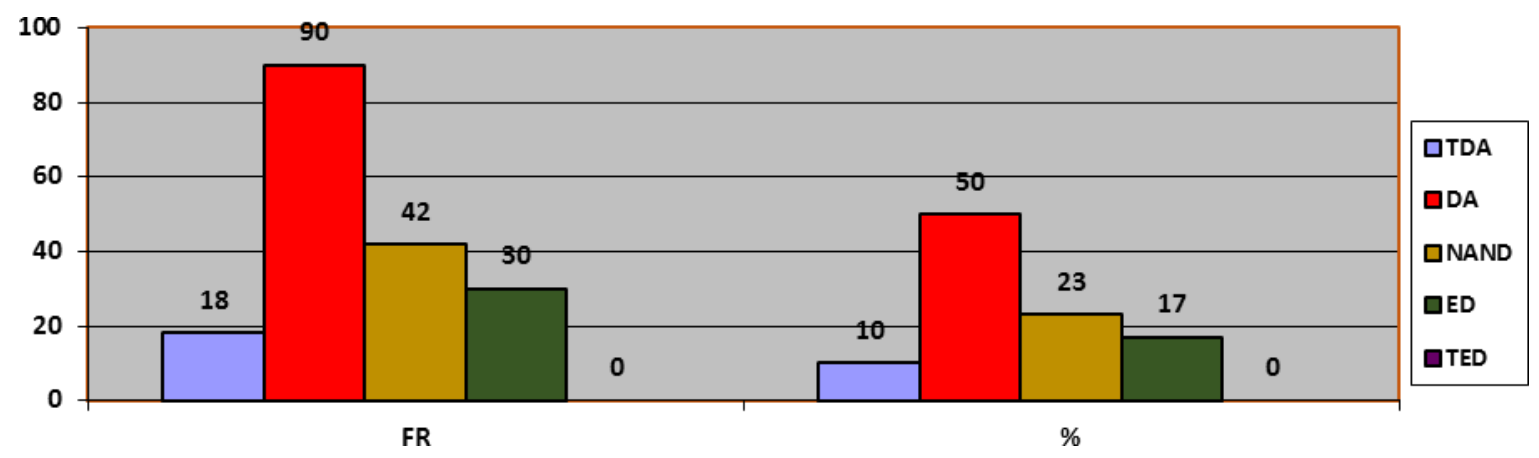

Fuente: Elaboración propia (2019)

En la tabla y gráfico 2, se presenta el análisis descriptivo del indicador nivel de coincidencia, en la que se indica que el $50 \%$ de los usuarios investigados están en desacuerdo entre lo dicen que hacen en la empresa con el servicio real que prestan. Seguido del $23 \%$ que indico estar ni de acuerdo ni en desacuerdo. Los menores porcentajes se indicaron para las alternativas 
totalmente de acuerdo y de acuerdo con el $10 \%$ y $17 \%$ respectivamente. Se destaca el hecho, en la cual la alternativa totalmente en desacuerdo no presento opinión alguna.

Tabla 3. Análisis Descriptivo del Indicador Imagen Corporativa

\begin{tabular}{ccccc}
\hline ANALISIS/ CRITERIOS & $\boldsymbol{F R}$ & $\%$ & $\boldsymbol{F R A}$ & $\% \boldsymbol{A}$ \\
\hline Totalmente de acuerdo & 18 & 10 & 18 & 10 \\
\hline De acuerdo & 24 & 13 & 42 & 23 \\
\hline $\begin{array}{c}\text { Ni de acuerdo ni en } \\
\text { desacuerdo }\end{array}$ & 36 & 20 & 78 & 43 \\
\hline $\begin{array}{c}\text { En desacuerdo } \\
\text { Totalmente en desacuerdo }\end{array}$ & 12 & 7 & 180 & 100 \\
\hline
\end{tabular}

Fuente: Elaboración propia (2019)

Gráfico 3. Análisis descriptivo del indicador Imagen Corporativa

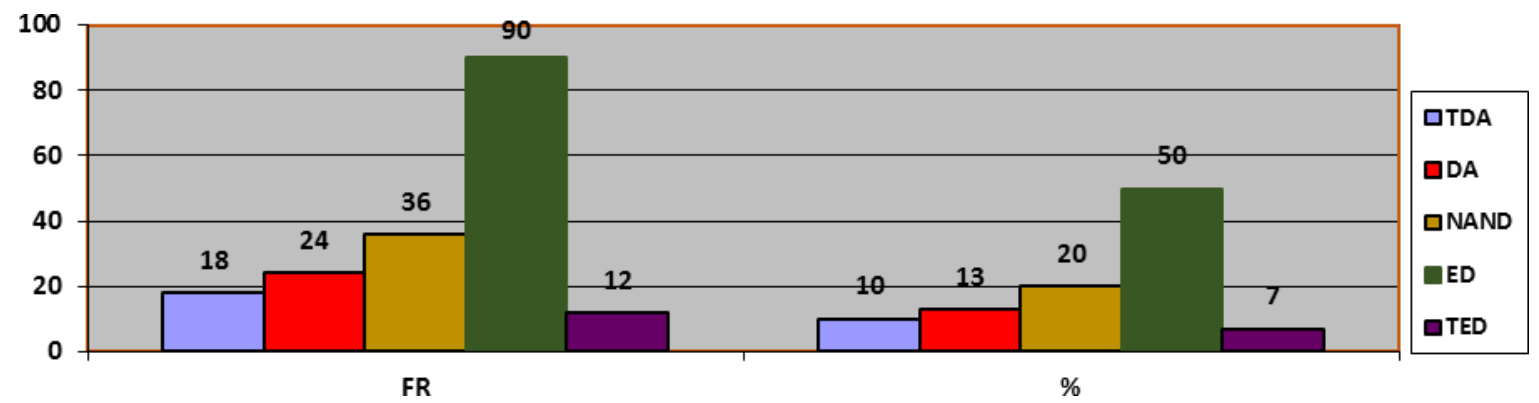

Fuente: Elaboración propia (2019)

En la tabla y gráfico 3, se presenta el análisis descriptivo del indicador Imagen Corporativa en la que se indica que el $50 \%$ de los usuarios investigados están en desacuerdo en que la empresa eléctrica en el cantón de Esmeraldas hace esfuerzos sistemáticos y conscientes con la finalidad de dar a conocer a sus clientes detalles de su cultura y elementos que la pueden distinguir de otros servicios que recibe la comunidad. Seguido del $20 \%$ que indico estar ni de acuerdo ni en desacuerdo. Los menores porcentajes se indicaron para las alternativas totalmente de acuerdo, de acuerdo y totalmente en desacuerdo con el 10\%, $13 \%$ y $7 \%$ respectivamente. 


\section{Conclusiones}

En atención a los resultados presentados se puede concluir que: Existe un ni acuerdo ni desacuerdo en que los usuarios del servicio eléctrico en Esmeraldas posean conocimiento de su estructura, cultura y organización de la misma, percibiendo que desde la institución no se considera como un valor agregado para su beneficio.

El relación al nivel de coincidencia, los investigados consideraron estar en desacuerdo con la adquisición de un enfoque global y correcta imagen de la empresa eléctrica, en tanto que no poseen una configuración que les permita poseer una imagen clara de la realidad del servicio que prestan a la comunidad del cantón de Esmeraldas.

En cuanto a la imagen corporativa, existe desacuerdo entre los investigados en cuanto a las relaciones de la empresa con la comunidad, específicamente en correspondencia a los valores propios de la región y su consideración para identificarse con una imagen positiva que le permita a sus empleados y usuarios satisfechos por pertenecer y/o utilizar sus servicios.

\section{Referencias}

1. Alba, J (2006). Comunicación y Conocimiento: Gestión del Desarrollo. España. Ediciones Simbióticas.

2. Aldana, J., \& Piña, J. (2017). Calidad del servicio prestado al cliente por los instructores de gimnasios. Revista Arbitrada Interdisciplinaria Koinonía, 2(3), 172-197. Recuperado de http://fundacionkoinonia.com.ve/ojs/index.php/revistakoinonia/article/view/59/46

3. Fernández, J. (2005).Sistemas organizacionales teoría y práctica. Bogotá D.C. Editorial universidad cooperativa de Colombia (educc).

4. Gutiérrez, F (2011). Pedagogía de la comunicación en la educación popular. Editorial Popular. S.A. Tercera edición. España.

5. Hernández, M. (2014). Estrategias de Relaciones Públicas para el posicionamiento de la imagen corporativa del sector público de salud marabino. Tesis de Grado. URBE. Maracaibo. 
6. Hernández, R; Fernández, C; y Baptista, P. (2014). Metodología de la Investigación. México. Editorial McGraw-Hill.

7. Herranz, J., Tapia, A., Lázaro, A. (2009). La Comunicación Interna en la Universidad. Investigar para conocer a nuestros públicos. RLCS Revista Latina de Comunicación Social. 64. Disponible en: http://www.revistalatinacs.org/

8. Mouriz, J (2007). Comunicación Estratégica y corporativa. Blog divulgativo sobre conceptos de comunicación corporativa. Disponible en: https://mouriz.wordpress.com/2007/06/04/comunicacion-estrategica/Multiciencias. Vol. 4. No 1. Punto Fijo

9. Sánchez, M; y Rincón, Y. (2009). Relaciones Públicas y Realidad Social. Artículo de Investigación Arbitrado Publicado. Revista Electrónica Razón y Palabra № 70. Año 14. Edición Especial de Relaciones Públicas. Noviembre 2009-Enero 2010. ISSN 1605-4806 Disponible http://www.razonypalabra.org.mx/14\%20S\%C3\%A1nchez_Rinc\%C3\%B3n.pdf. Consultado en Julio del 2015.

10. Tamayo y Tamayo, M. (2012). El Proceso de la Investigación Científica. Cuarta edición. México: Editorial Limusa, S.A.

11. Vilcarromero, M. (2009), Imagen positiva. Gestión estratégica de la imagen de las empresas. Madrid: Editorial Pirámide.

\section{References}

1. Alba, J (2006). Communication and Knowledge: Development Management. Spain. Symbiotic editions. 
2. Aldana, J., \& Piña, J. (2017). Quality of service provided to the client by gym instructors. Interdisciplinary Arbitrated Review Koinonía, 2 (3), 172-197. Recovered from http://fundacionkoinonia.com.ve/ojs/index.php/revistakoinonia/article/view/59/46

3. Fernández, J. (2005). Organizational systems theory and practice. Bogotá D.C. Editorial cooperative university of Colombia (educc).

4. Gutiérrez, F (2011). Pedagogy of communication in popular education. Popular Publishing. S.A. Third edition. Spain.

5. Hernández, M. (2014). Public Relations Strategies for positioning the corporate image of the public health sector in Marabino. Thesis. CITY. Maracaibo

6. Hernández, R; Fernández, C; and Baptista, P. (2014). Investigation methodology. Mexico. McGraw-Hill editorial.

7. Herranz, J., Tapia, A., Lázaro, A. (2009). Internal Communication in the University. Investigate to meet our audiences. RLCS Latin Journal of Social Communication. 64. Available at: http://www.revistalatinacs.org/

8. Mouriz, J (2007). Strategic and corporate communication. Informative blog about corporate communication concepts. Available https://mouriz.wordpress.com/2007/06/04/comunicacion-estrategica/Multiciencias. Vol.

\section{No. 1. Fixed Point}

9. Sánchez, M; and Rincon, Y. (2009). Public Relations and Social Reality. Published Arbitrated Research Article. Electronic Magazine Reason and Word No. 70. Year 14. Special Edition of Public Relations. November 2009-January 2010. ISSN 1605-4806 Available http://www.razonypalabra.org.mx/14\%20S\%C3\%A1nchez_Rinc\%C3\%B3n.pdf. Consulted in July 2015. 
